\title{
Davide Schiffer: Attraverso il Microscopio
}

\author{
Ezio Giacobini
}

Published online: 6 July 2011

(C) Springer-Verlag 2011

This book represents much more than the personal history of an internationally renowned Italian neuropathologist and neuroscientist. It is not only about what he has seen through the lens of the microscope during half a century of clinical and laboratory research. It also explores the basis of clinical reasoning, the perception and the emotions of inspecting the microscopic world, and the language and aesthetic values of that world.

Neuropathology developed in the nineteenth century almost simultaneously in France, England and Germany. However, the question of to whom this discipline belongs is still open. Neurologists, psychiatrists, and pathologists all claim it as their own. At the end the pathologists, at least in Italy, won.

According to Schiffer, "the value of a brain without a clinical record is equal to the value of clinical record without a brain." This underlines the critical importance of combining clinical and pathological knowledge. The author emphasizes the fact that the use of the microscope does not simply represent "a technique" but represents a "true dialectic moment between the observer and the object to be observed."

The subjective perception of the observer vis-à-vis the object may influence the identification and recognition process and, at the end, condition the diagnosis. This complex process is not detached from the observer's emotions and feelings which may inform his conclusions. It is, therefore, important, in order to "reach a total immersion in the microscopic world," to devote one's undivided attention, being alone and isolated from one's own world

E. Giacobini $(\bowtie)$

Department of Internal Medicine, Rehabilitation and Geriatrics, University of Geneva Medical School, Geneva, Switzerland e-mail: Ezio.Giacobini@ @cuge.ch (no human voices, possibly very low classic music!). The neuropathologist at the microscope has to find his way through the intricate structure of the nervous system, to explore a certain path "not hesitating to look again and again". It is like "swinging slowly in a dense fluid trying to catch all details".

It is also important "to know in advance" what one wants to see, keeping in mind the clinical record of the specific case, before sitting in front of the microscope. The pathologist armed with only the slide is, in important ways, blind without knowledge of the patient.

A histological slide has its own intrinsic aesthetic and dramatic value, expressed by the designs and the colors of the specimen. Who could not agree with this opinion looking at a silver-impregnated slide of the human cortex designed by hand by Ramón y Cajal? According to the author, even a specimen from a malign tumor may exhibit aesthetic beauty. He uses the example of a section of the aggressive Burkitt lymphoma, appearing as a "starry sky." The sight of apoptosis, the "after the fall catastrophic image", is a representation of the announced death of a cell. A group of apoptotic dead cells appears as the "feullies muertes" of the tissue.

The dying nerve cell tells us about its tragic story as a victim of "neuronophagia" i.e., being eaten up by the surrounding satellite cells. A picture of the dying neuron which resembles the statue of the "dying slave" of Michelangelo moves one to piety. In reality, the satellite cells do not eat up the neuron. Instead, they simply help it die.

Classic histology is now supplemented with other more sophisticated techniques such as histochemistry, immunohistochemistry using a full array of antibodies, and by fluorescence microscopy. With laser technology, resolution has reached the sub-cellular level. 
Quantitative cytochemistry allows one to exactly measure substrates, enzymes, small proteins, and peptides in one single cell. Such developments have presented the neurologist-neuropathologist with a new dilemma: To become a molecular analyst, to continue as a clinician, or to combine both? The author himself has chosen the solution of becoming a molecular biologist while maintaining close contact with clinical problems. Undoubtedly, this is one of the hardest and less common ways. Instead, most choose to establish a close collaboration with molecular biologists and forego the closer historic links of laboratory to clinical ward.

The book clearly demonstrates that the game of neurological oncology is not a "solo" performance but has four players: The neurosurgeon, the neuropathologist, the neurooncologist and the neuroradiologist. The "perfect neurosurgeon" of the past-the one who every afternoon following surgery went to the neuropathology laboratory and sat at the microscope-is rare today, as is the neurologist or neurosurgeon who attends at his or her patient's autopsy.

At the end, the pathologist is left alone in his laboratory with the uncertainties: What is a normal tissue and what is not? Normality is what, within given certain limits, is statistically more frequent while abnormality is what falls outside these subjective limits. According to the author, microscopic world and real world do not conflict but integrate with each other. In science, they represent two facets of the same reality which can be explored either by looking at thousands of cells in a cortical section of a human brain as the histologist does, or by the electrophysiologist who examines one by one the few cells constituting the whole brain of a sea slug, such as the aplysia model of Kandel.

The scientific observations reported in this book are enriched and mixed with the personal history of the author: His difficult youth during and after the war as the son of a deported father who never returned from a Nazi concentration camp, his long and emotional visit to postwar Germany, and his experiences in the laboratory of the most famous couple of German neuropathologists, Cecile and Oskar Vogt.

The story of such a long career in academia is full of inspiring historical figures, from Linnaeus, the methodic analyst and founder of systematic botany, to the controversial figure of Lombroso, one of the fathers of biological positivism. Philosophical thinking is present throughout the book, from Cartesius to Kant, from Locke to Russell and from Popper to Kuhn.

These varied ingredients combine to make up a fascinating book that takes the reader through the microscopic universe, a marvelous trip that allows each of us to accompany Alice into the author's microscopic Wonderland. 